\title{
Eigenconjugation: An Approach for Computing Image Similarity
}

\author{
V. Ortega-González, A. Angeles-Yreta, J. Medina-Apodaca, \\ V. Landassuri-Moreno, and J. Figueroa-Nazuno \\ Centro de Investigación en Computación, Instituto Politécnico Nacional \\ Unidad Profesional "Adolfo López Mateos", 07279, México, D.F. \\ eortegag631@ipn.mx, malberto@sagitario.cic.ipn.mx, jmmedinaa@ipn.mx, \\ victorm@sagitario.cic.ipn.mx, jfn@cic.ipn.mx
}

\begin{abstract}
We introduce a method for computing similarity between two square matrices based on the information given by their eigenvalues and eigenvectors. The idea is to evaluate the effect of the conjugation of the original matrices and the eigenvectors and eigenvalues of each other. Then, we exemplify its utility for computing similarity between square images from a classified bank of pictures. The performance of the method is evaluated with diverse experiments.
\end{abstract}

\section{Introduction}

Comparing matrices is an interesting open problem in computer sciences. Moreover, since matrices are a very useful representation for many kinds of data, it has a lot of applications in many different fields. On the conventional paradigm [1], measuring similarity between entities implies the selection of those properties considered as essential. These subjective elections are commonly erroneous and always incomplete. In many cases, the best techniques are those that use all the information available of the involved entities. For the specific problem of comparing matrices, we should prefer a method that works with all the information contained in each element of the bi-dimensional array.

In Linear Algebra, eigenvalues and eigenvectors are often used to characterize a transformation defined by a square matrix [6]. The determination of those vectors and values is a very well studied problem, and there are a lot of numerical processes which aim to solve it.

Definition 1. Given a linear transformation $M$ (defined by a square matrix) from $\Upsilon$ into $\Upsilon$, a nonzero vector $v$ in $\Upsilon$ is an eigenvector of $M$ if

$$
M v=\lambda v
$$

where $v \neq \check{0}$ and $\|v\|_{2}=1$.

The scalar $\lambda$ is called the eigenvalue of $M$ corresponding to $v[3$. Thus, we could say that an eigenvector of a square matrix is a non-null vector whose direction 
is unchanged by the transformation and that the factor by which the magnitude is scaled is called the eigenvalue of that vector.

A square matrix $M$ of $n \times n$ has up to $n$ distinct pairs formed by an eigenvalue and a normalized eigenvector: $M v^{(1)}=\lambda_{1} v^{(1)}, M v^{(2)}=\lambda_{2} v^{(2)}, \cdots$, $M v^{(n)}=\lambda_{n} v^{(n)}$; where $\left\|v^{(i)}\right\|_{2}=0$ for $i=1,2, \cdots, n$. It is common to represent the eigenvalues of a square matrix into a diagonal matrix called $\Lambda$, and the normalized eigenvectors are often represented as the columns of a square matrix called $V$, where they occupy their position according to $\Lambda$ as follows

$$
\begin{gathered}
\Lambda=\left[\begin{array}{cccc}
\lambda_{1} & 0 & \cdots & 0 \\
0 & \lambda_{2} & \cdots & 0 \\
\vdots & & \ddots & \vdots \\
0 & 0 & \cdots & \lambda_{n}
\end{array}\right] \\
V=\left[\begin{array}{llll}
v^{(1)} & v^{(2)} & \cdots & v^{(n)}
\end{array}\right]
\end{gathered}
$$

It is useful to condense all the information given by the eigenvalues and eigenvectors into a single algebraic expression

$$
M V=V \Lambda
$$

It is mathematically accepted to say that two $(n \times n)$ matrices $A$ and $B$ are similar if there is an invertible matrix $P$ for which $B=P^{-1} A P[3$. This is an equivalence relation on $\Re_{n \times n}$. We also know that if $A$ and $B$ are mathematically similar, they share the same eigenvalues. But this idea is inadequate for real cases where this relation does not describe sufficiently the data. Together, the eigenvectors and eigenvalues describe completely and univocally the intrinsic information of a square matrix. Thus, it is not possible to find two distinct matrices with the same eigenvalues and eigenvectors. We could take advantage of this unique characterization in order to represent a matrix with neither loss of information nor any kind of ambiguity.

\section{Eigenconjugation of Two Square Matrices}

Definition 2. Given two square matrices $(n \times n) A$ and $B$ with the matrices of eigenvalues $\Lambda_{A}, \Lambda_{B}$ and the matrices of eigenvectors $V_{A}, V_{B}$ respectively, we know it is true that

$$
\begin{aligned}
& V_{A}=A V_{A} \Lambda_{A}^{-1} \\
& V_{B}=B V_{B} \Lambda_{B}^{-1}
\end{aligned}
$$

where $A, B, \Lambda_{A}, \Lambda_{B}, V_{A}, V_{B} \in \mathbb{C}_{(n \times n)}$. Then, the matrices $V_{A}^{\prime}$ and $V_{B}^{\prime}$ know as the eigenconjugation of $A$ and $B$ are defined as

$$
\begin{aligned}
& V_{A}^{\prime}=B V_{A} \Lambda_{A}^{-1} \\
& V_{B}^{\prime}=A V_{B} \Lambda_{B}^{-1}
\end{aligned}
$$

where $V_{A}^{\prime}, V_{B}^{\prime} \in \mathbb{C}_{(n \times n)}$. 
Since $V_{A}$ and $V_{B}$ are matrices composed by column vectors, we considered $V_{A}^{\prime}$ and $V_{B}^{\prime}$ to have the same structure. Therefore, the column vectors of $V_{A}^{\prime}$ express the effect of the conjugation of $B$ with the eigenvalues and eigenvectors of $A$. And the columns vectors of $V_{B}^{\prime}$ express the effect of the conjugation of $A$ with the eigenvalues and eigenvectors of $B$.

\section{Evaluation of the Eigenconjugation as a Similarity Measure for Square Matrices}

In this section we introduce a new method which aims to measure an approximated distance between two square matrices based on the algebraic conjugation of the original matrices and the eigenvalues and eigenvectors of each other.

It is possible to represent the distances of each of the column vectors from $V_{A}$ to $V_{A}^{\prime}$ into a sequence of $n$ elements, and the distances from $V_{B}$ to $V_{B}^{\prime}$ into another sequence. An interesting assumption is that if the original matrices $A$ and $B$ are similar, then the behaviours of both sequences are also similar. Now, we define a new matrix operator for determinining the distances between those vectors.

Definition 3. Given two matrices $(n \times m) U$ and $V$, both composed by column vectors such as

$$
\begin{aligned}
& U=\left[\begin{array}{lll}
u^{(1)} & u^{(2)} \cdots u^{(m)}
\end{array}\right] \\
& V=\left[\begin{array}{lll}
v^{(1)} & v^{(2)} \cdots v^{(m)}
\end{array}\right]
\end{aligned}
$$

the operator $*$ is defined as

$$
U * V=\left[e\left(u^{(1)}, v^{(1)}\right) e\left(u^{(2)}, v^{(2)}\right) \cdots e\left(u^{(m)}, v^{(m)}\right)\right]
$$

where $e(u, v)$ calculates the Euclidean distance between vectors $u$ and $v$

$$
e(u, v)=\left(\sum_{i=1}^{n}\left(u_{i}-v_{i}\right)^{2}\right)^{\frac{1}{2}} .
$$

The result is a sequence that contains the distances between the consecutive pairs of columns vectors from both matrices. Now, we define two useful sequences

$$
\begin{aligned}
& d_{1}=V_{A} * V_{A}^{\prime} \\
& d_{2}=V_{B} * V_{B}^{\prime}
\end{aligned}
$$

Therefore $d_{1}$ express the Euclidian distances between each of the vectors of $V_{A}$ and $V_{A}^{\prime}$, and $d_{2}$ express the Euclidian distances between each of the vectors of $V_{B}$ and $V_{B}^{\prime}$. Both sequences characterize the effect of the eigenconjugation of $A$ and $B$. The characteristic sequences of an Eigenconjugation have always an uptrend.

The hypothesis is that the original matrices $A$ and $B$ are as similar as sequences $d_{1}$ and $d_{2}$ are: 


$$
A \approx B \rightarrow d_{1} \approx d_{2}
$$

Since the expression (15) is an one-way logical implication, we must assume that $A$ and $B$ are matrices that belong to the same class.

There are many techniques for comparing sequences as time series. For this work we chose Dynamic Time Warping (DTW) for minimize the base distances between these sequences. For more information we suggest to see [4].

Definition 4. Given two sequences $Q=\left\langle q_{1}, q_{2}, \cdots, q_{n}\right\rangle$ and $C=\left\langle c_{1}, c_{2}\right.$, $\left.\cdots, c_{m}\right\rangle$, the dynamic time warping distance DTW is defined recursively as follows [2]:

$$
\begin{gathered}
D T W(\langle\rangle,\langle\rangle)=0 \\
D T W(Q,\langle\rangle)=D T W(\langle\rangle, C)=\infty \\
D T W(Q, C)=d_{\text {base }}(f(Q), f(C))+\min \left\{\begin{array}{c}
D T W(Q, r(C)) \\
D T W(r(Q), C) \\
D T W(r(Q), r(C))
\end{array}\right.
\end{gathered}
$$

where

$$
d_{\text {base }}\left(q_{i}, c_{j}\right)=\left|q_{i}-c_{j}\right|
$$

and $f\left(\left\langle p_{1}, p_{2}, \cdots, p_{k}\right\rangle\right)=p_{1}, r\left(\left\langle p_{1}, p_{2}, \cdots, p_{k}\right\rangle\right)=\left\langle p_{2}, p_{3}, \cdots, p_{k}\right\rangle$.

It is assumed that $Q$ and $C$ are the same if $\operatorname{DTW}(Q, C)=0$, they are similar when $D T W(Q, C) \rightarrow 0$, and they are different when $D T W(Q, C) \rightarrow \infty$.

For the experimental results we noticed that the numeric value given by the computation of $D T W$ could be affected by the scale of the magnitudes of the original matrices. So there were many near false positives cases when we compare one image to a bank of hundreds. Therefore we modified (19) in order to employ a kind of normalization similar to the one occupied in Normalized Root Mean Square Error (NRMS) measure. Thus, $d_{\text {base }}$ is redefined as follows:

$$
d_{\text {base }}\left(q_{i}, c_{j}\right)=\left|\frac{q_{i}-c_{j}}{\bar{q}-q_{i}}\right|
$$

where $\bar{q}$ is the mean value of the fist sequence. Therefore $D T W(Q, C) \neq$ $D T W(C, Q)$ in general. For the implementation we used an optimized version of $D T W$ know as Fast Dynamic Time Warping ( $F D T W$ ), which is a nonrecursive approximation of computational complexity less than a polynomial of degree 2.

Definition 5. Given two square matrices $A$ and $B$ of the same class, the similarity measure $\operatorname{sim}(A, B)$ is defined as

$$
\operatorname{sim}(A, B)=\sqrt{D T W^{2}\left(d_{1}, d_{2}\right)+D T W^{2}\left(d_{2}, d_{1}\right)}
$$

where $d_{1}$ and $d_{2}$ are the characteristic sequences of the eigenconjugation of $A$ and $B$. 
In the following figures we show four examples of comparisons between pairs of same-class images. In Figure 1 it is possible to appreciate by simple glance, the relation of similarity supposed by (15). In the first case both sequences have a similar behaviour and the original images are also alike. In the second case it is observable the opposite situation.

(a)

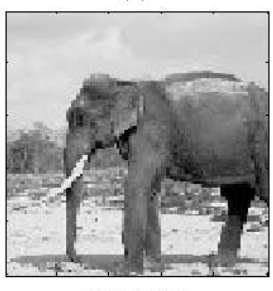

A [img.592]

(d)

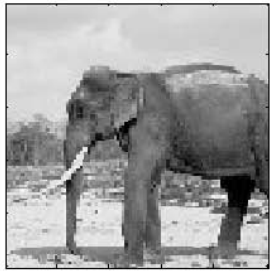

A [img.592] (b)

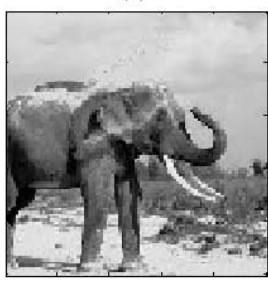

B [img.589]

(e)

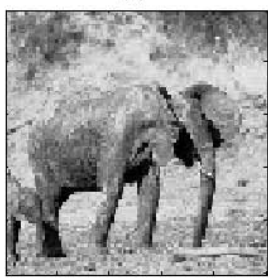

C [img.558] (c) $\operatorname{sim}(A, B)=38.6907$

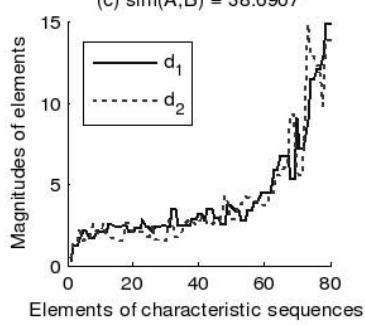

(f) $\operatorname{sim}(A, C)=462.9554$

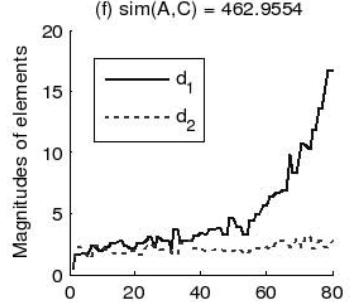

Elements of characteristic sequences

Fig. 1. Image $\mathrm{A}$ is compared to $\mathrm{B}$ and their characteristic sequences $-d_{1} \& d_{2^{-}}$are showed in (c). Image A is also compared to C, their sequences $-d_{1} \& d_{2}$ - are showed in (f). All images are of size $100 \times 100$ pixels. Only the first $80 \%$ of the elements of the sequences are plotted. Codes in brackets are the references of the images.

In Figure 2 two more experiments are showed, where it is important to notice that the numeric values given by sim are validated by the similarity relation of the original images.

\section{Comparing Square Images Using Eigenconjugation}

Although humans are capable of a complex and efficient performance while comparing images, this task is quite difficult for automated systems. Eigenconjugation validates the similarity relation between data matrices using numerical processes that can be easily implemented into a computer.

Although there are many techniques for pre-processing images such as filtering or thresholding, we rather work with the original information without any other stage or process. This is necessary because in this paper we introduce a method for comparing any kind of square matrices, not only for the specific case of square images. 
(a)

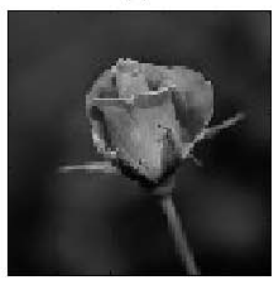

A [img.681]

(d)

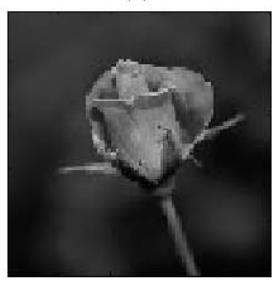

A [img.681] (b)

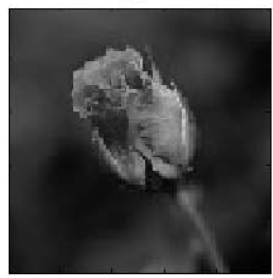

B [img.682]

(e)

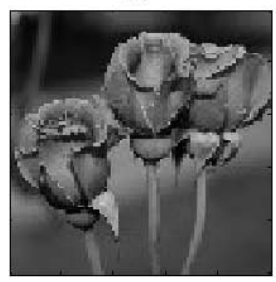

C [img.683] (c) $\operatorname{sim}(A, B)=72.8811$

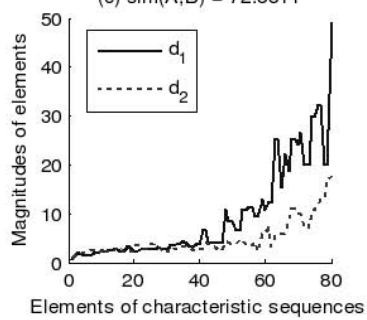

(f) $\operatorname{sim}(A, C)=3186.4243$

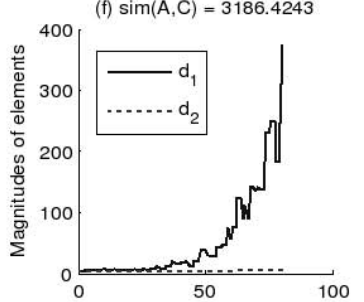

Elements of characteristic sequences

Fig. 2. Image $A$ is compared to $B$ and their characteristic sequences $-d_{1} \& d_{2}$ - are showed in (c). Image $\mathrm{A}$ is also compared to $\mathrm{C}$, their sequences $-d_{1} \& d_{2^{-}}$are showed in (f). All images are of size $100 \times 100$ pixels. Only the first $80 \%$ of the elements of the sequences are plotted. Codes in brackets are the references of the images.

Using eigenconjugation, a comparison of two square matrices $(n \times n)$ has a computational complexity of somewhat less than $4 n^{3}+12 n^{2}+8 n$ field operations; where the following stages are considered: algebraic eigenconjugation, construction of the characteristic sequences and the evaluation with FDTW.

Given a set of matrix representations of same-class images $(\Pi)$ the procedure for sorting the elements by its similarity with a query is as follows (it is supposed that all images are square normalized and have the same size):

1. Select the query matrix $A \mid A \in \Pi$.

2. Calculate the eigenvectors and eigenvalues of $A$.

3. For each matrix $B$ on the set $(B \mid B \in \Pi)$ :

(a) Calculate the eigenvectors and eigenvalues of $B$.

(b) Obtain the eigenconjugation sequences of $A$ and $B$ : $d_{1}$ and $d_{2}$.

(c) Calculate the similarity measure $\operatorname{sim}(A, B)$; and save the result into a list.

4. Sort the list and show the results.

At first we worked with simple matrices obtained for the grayscale representation of the images. Obviously the information given by grayscale matrices is not enough for differencing efficiently each image from others. During these first experiments the results were not very satisfactory. It was necessary to include the information given by the colour components. Since the original images were available in RGB format, we decided to make a triple comparison using the matrices of the red, green and blue components. So there were three distances for 
each comparison. These three values were the components of a vector of distance whose absolute magnitude was defined as the total distance between the coloured matrices. Thus the results were sensibly improved in almost all the cases. But there were still some inconsistencies.

The best results were obtained when we used a unique jointly-coloured matrix representation of each image. This coloured matrix is constructed by an alternative permutation of the bits of the three colour components of each pixel. Thus, one single numeric value is obtained for each pixel. In the former representations the interval for pixel values was small $(0-255)$, with the use of coloured matrices the interval or variability is incremented (up to $256^{3}$ ). Therefore the amount of available information is extended, and the process of similarity measuring is improved.

Every RGB image could be decomposed into three component matrices

$$
R=\left(r_{i j}\right) \quad G=\left(g_{i j}\right) B=\left(b_{i j}\right)
$$

where each element $(r, g$ and $b)$ of the matrices has a binary representation as

$$
\begin{gathered}
r=\left(r_{7} r_{6} r_{5} r_{4} r_{3} r_{2} r_{1} r_{0}\right)_{2} \\
r=\sum_{k=0}^{7} 2^{k} \cdot r_{k}
\end{gathered}
$$

where $r_{k} \in\{0,1\}$ for $0 \leq k \leq 7$.

Definition 6. Given a square RGB image $(n \times n)$, its jointly-coloured representation $M$ is defined as follows:

$$
M=C(R, G, B)
$$

where $M=\left(m_{i j}\right)$; and each element $m$ of $M$ has the following binary composition

$$
\begin{gathered}
m=\left(r_{7} g_{7} b_{7} r_{6} g_{6} b_{6} \cdots r_{0} g_{0} b_{0}\right)_{2} \\
r=\sum_{k=0}^{7} 2^{3 k+2} \cdot r_{k}+2^{3 k+1} \cdot g_{k}+2^{3 k} \cdot b_{k}
\end{gathered}
$$

where $r_{k}, g_{k}, b_{k} \in\{0,1\}$ for $0 \leq k \leq 7$.

This representation prevents us for giving and excessive importance to a specific colour component. Nevertheless there is still an undesirable minimized favouritism. The defined order $(\mathrm{r}-\mathrm{g}-\mathrm{b})$ in the combination is arbitrary, and it could be changed for optimizing the results in specific cases.

We tried experimentally to use normalization in different steps of the procedure, but the results did not improve except for some specific cases. General applications should not use normalization. 


\section{Experimental Results}

In the following figures we illustrate some experiments where a query image was given, and the five nearest coloured images are showed (among more than 100 elements in each class). In all cases the jointly-coloured matrix representation was used. These results are very interesting and precise although they have to be validated by human inspection. Thus, there is no way to make a rigid objective evaluation of the effectiveness of the method.

In Figure 3, the two more similar images were placed next to the target. The other results could be irrelevant or appear to be erroneous. But the experiment is good because it brought the most similar elements at first.
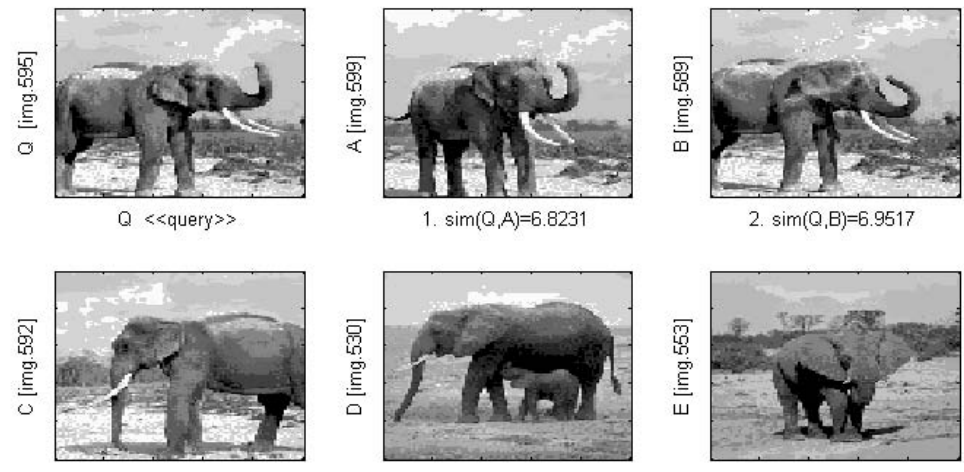

4. $\operatorname{sim}(Q, D)=7.9689$

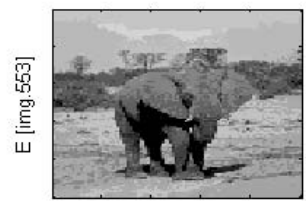

5. $\operatorname{sim}(\mathrm{Q}, \mathrm{E})=8.2632$

Fig. 3. Searching over the Elephant class. Image Q "query" was compared to all the elements from the class. Images A to $\mathrm{E}$ are the nearest found specimens given by the sim measure. Codes in brackets are the corresponding references of the images.

Two experiments among the Horse class are showed in Figure 4 and Figure 5 These results are very appropriate for exemplify the correct performance of the method. They are good because the Horse class is correctly classified and the set is homogeneous. Therefore the method could determine with high precision the similarity over an homogeneous set.

Every class of images has a degree of homogeneity which is merely a subjective estimation of resemblance among its elements. In Figure 6 the results are very acceptable; in fact they are almost exactly. It is important to observe that the method is not infallible, that its efficiency depends strongly on the degree of homogeneity between the elements of the class.

\section{Conclusions and Future Work}

Eigenconjugation is a method for computing similarity between same-class images. And this might be a difficult task because these images are already similar. 


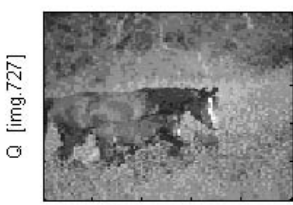

$Q<$ Q query $>>$

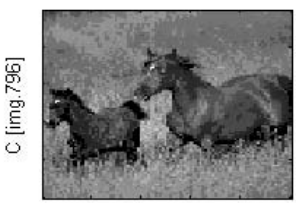

3. $\operatorname{sim}(Q, C)=8.6562$

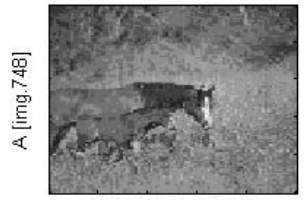

1. $\operatorname{sim}(Q, A)=6.3166$

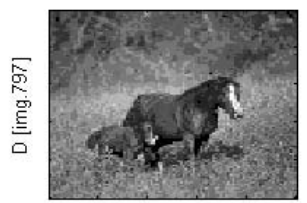

4. $\operatorname{sim}(Q, D)=9.228$

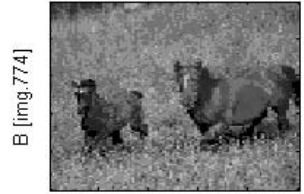

2. $\operatorname{sim}(Q, B)=8.6135$

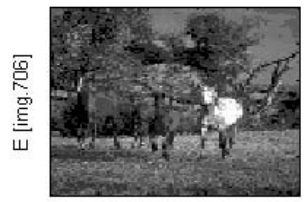

5. $\operatorname{sim}(\mathrm{Q}, \mathrm{E})=10.664$

Fig. 4. Searching over the Horse class. Images A to E are the nearest specimens of Q given by the sim measure. Codes in brackets are the references of the images.

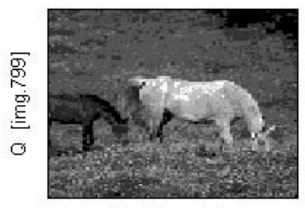

$Q<$ query $>$

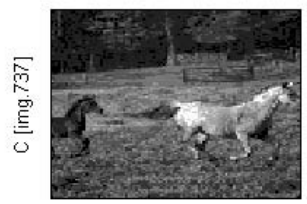

3. $\operatorname{sim}(Q, C)=18.1704$

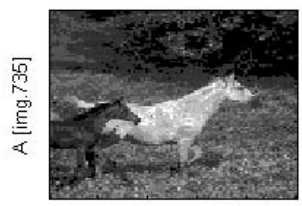

1. $\operatorname{sim}(\mathrm{Q}, \mathrm{A})=16.4143$

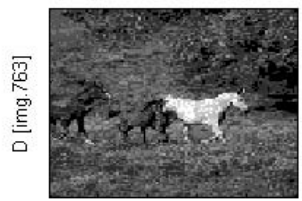

4. $\operatorname{sim}(Q, D)=18.358$

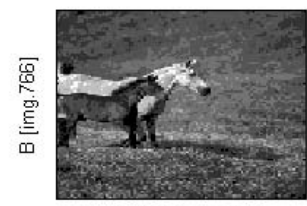

2. $\operatorname{sim}(Q, B)=18.1224$

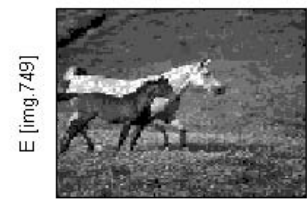

5. $\operatorname{sim}(Q, E)=19.6069$

Fig. 5. Searching once again over the Horse class. Images A to $E$ are the nearest specimens of Q given by sim. Codes in brackets are the references of the images.

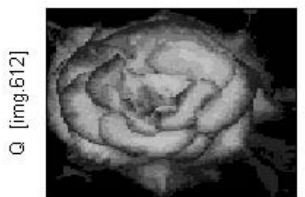

$Q<$ query $>$

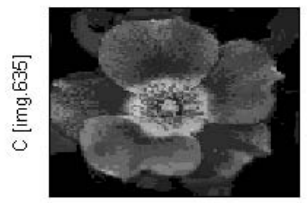

3. $\operatorname{sim}(Q, C)=171.1386$

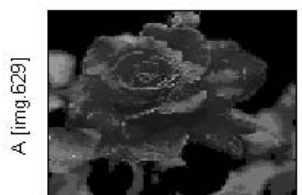

1. $\operatorname{sim}(Q, A)=107.9087$

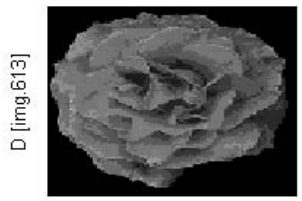

4. $\operatorname{sim}(Q, D)=195.8044$

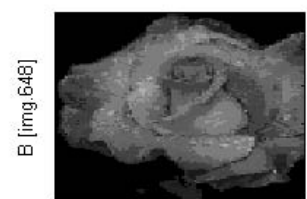

2. $\operatorname{sim}(Q, B)=167.4284$

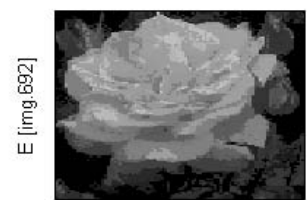

5. $\operatorname{sim}(Q, E)=197.5045$

Fig. 6. Searching over the Flower class. Images A to E are the nearest specimens of Q given by the sim measure. Codes in brackets are the references of the images. 
The proposed method could determine with high precision the similarity over an homogeneous set. Its efficiency depends strongly on the degree of homogeneity between the elements of the class. We have evaluated the method using matrix representations of colour images having better results while working with homogeneous sets. Nevertheless, this method could be applied for every kind of square data matrices. The method evaluates the similarity based on the whole structure of the matrices. In this paper we have discussed about its limitations and characteristics in different cases of use.

Eigenconjugation is a new different approach for computing image similarity. Some other methods [8] need to first process all the elements of the class, but Eigencojugation could evaluate the distance only between two matrices without any information from the other elements. A profound comparison with other techniques is a complicated task which is out of the reach of this paper.

Some future work suggestions are:

1. Using other similarity techniques and measures for time series.

2. Using eigenconjugation for computing similarity with other kinds of data matrices.

3. Complementing eigenconjugation with a classification method for working with non same-class images.

4. Comparing eigenconjugation with other techniques for image similarity.

Acknowledgments. The authors would like to thank the National Polytechnic Institute (IPN) and the National Council for Science and Technology (CONACyT) for supporting young people in academic activities.

\section{References}

1. J. Figueroa-Nazuno, A. Angeles-Yreta, J. Medina-Apodaca, V. Ortega-González, K. Ramírez-Amaro, M. Mirón-Bernal and V. Landassuri-Moreno: "Sobre el problema de Semejanza", Reportes Técnicos, CIC-IPN, México, 2006.

2. Sang-Wook Kim: "An Index-Based Approach for Similarity Search Supporting Time Warping in Large Sequence Databases", IBM T.J. WRC, ICDE 2001.

3. Brinkmann-Klotz: "Linear Algebra and Analytic Geometry", Addison-Wesley Publishing Company, 1971.

4. L. Rabiner and B.-H. Juang: "Fundamentals of Speech Recognition", Prentice Hall, 1993.

5. E. Keogh: "Exact Indexing of Dynamic Time Warping", in Proceedings of the 28th VLDB Conference, pp. 406-417, 2002.

6. J. Medina-Apodaca, J. Figueroa-Nazuno and S. García: "Time Series modeling using Recurrence Plots and Face Recognition techniques", Congreso Nacional de Física 2002, México, 2002.

7. A. Angeles-Yreta and J. Figueroa-Nazuno: "Computing Similarity Among 3D Objects Using Dynamic Time Warping", 10th Iberoamerican Congress on Pattern Recognition, Cuba, 2005.

8. Pentland, T. Starner, N. Etcoff, A. Masoiu, O. Oliyide and M. Turk: "Experiments with Eigenfaces", International Joint Conference on Artificial Intelligence 1993, Chamberry, France, 1993. 